\title{
Identification of Mest/Peg1 gene expression as a predictive biomarker of adipose tissue expansion sensitive to dietary anti-obesity interventions
}

\author{
Anja Voigt ${ }^{1} \cdot$ Joan Ribot $^{2} \cdot$ Agustín G. Sabater $^{2} \cdot$ Andreu Palou $^{2} \cdot$ M. Luisa Bonet ${ }^{2}$ • \\ Susanne Klaus ${ }^{1}$
}

Received: 17 March 2015/Accepted: 17 June 2015/Published online: 5 July 2015

(C) Springer-Verlag Berlin Heidelberg 2015

\begin{abstract}
Food components with anti-obesity properties are commonly evaluated using mouse models of diet-induced obesity. The ability of these components to reduce or prevent white adipose tissue (WAT) accumulation is usually tested in feeding trials of several weeks duration in order to detect significant effects on fat mass expansion. Here, we aimed to identify early, predictive biomarkers for WAT expansion. We performed a 5-day high-fat diet (HFD) feeding trial with C57BL/6J mice using different established anti-obesity interventions: epigallocatechin gallate, replacing dietary lipids by n-3 PUFA, and increasing dietary protein. WAT gene expression was analyzed of genes known to be similarly affected by shortand long-term HFD. Gene expression of Leptin and Mest (mesoderm-specific transcript) was increased by HFD and
\end{abstract}

Anja Voigt and Joan Ribot have contributed equally to this work.

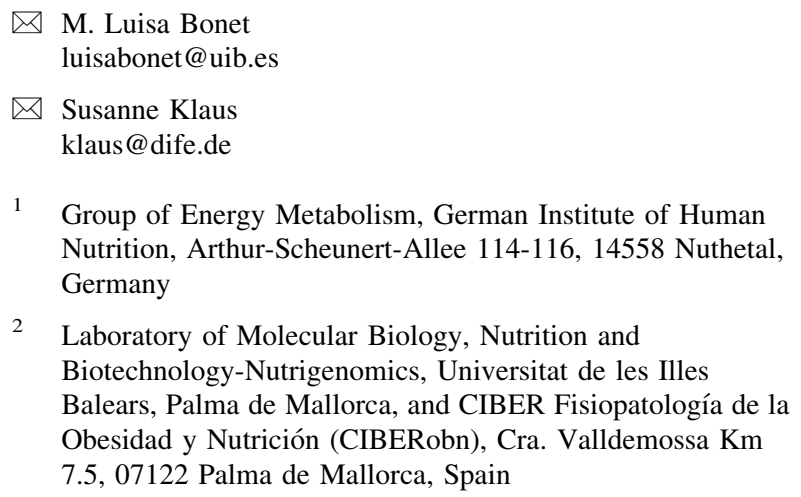

2 Laboratory of Molecular Biology, Nutrition and Biotechnology-Nutrigenomics, Universitat de les Illes Balears, Palma de Mallorca, and CIBER Fisiopatología de la Obesidad y Nutrición (CIBERobn), Cra. Valldemossa Km 7.5, 07122 Palma de Mallorca, Spain

normalized by all anti-obesity interventions. In a second experiment, translatability to whole blood-based expression data was assessed. Mice were challenged for 21 days with a HFD without or with simultaneous treatment with anti-obesity bioactives, hydroxytyrosol or resveratrol, and compared for parameters including Leptin and Mest expression in whole blood at day 5. While Leptin mRNA could not be detected in mouse whole blood, there was an induction of Mest mRNA by HFD which was suppressed by hydroxytyrosol. Moreover, Mest expression in whole blood at day 5 positively correlated with adiposity and negatively with lean body mass and the subcutaneous/visceral fat ratio at day 21 . We conclude that gene expression of Leptin and Mest in WAT and of Mest in whole blood represent early, predictive markers of adipose tissue expansion of potential usefulness in nutritional studies and trials.

Keywords Obesity - Whole blood - Mesoderm-specific transcript (Mest/Pegl) · Epigallocatechin gallate (EGCG) · n-3 PUFA · High-protein diet · Hydroxytyrosol ·

Resveratrol

\section{Introduction}

Obesity prevalence is increasing worldwide not only in industrialized but also in developing and transition countries, and nearly three million deaths each year are obesity related (Scully 2014). One approach in combatting obesity is the use of dietary interventions consisting of altered dietary nutrient composition or supplementation of bioactive ingredients in order to prevent and/or treat obesity. For an effective development of this approach, early biomarkers of adipose tissue expansion that are both 
predictive of fat accumulation in the long-term and sensitive to dietary anti-obesity interventions are of great interest, as their availability can, in the first place, expedite the screening and evaluation of candidate ingredients/conditions in animal trials. Of particular interest are biomarkers of this type derived from easily accessible, noninvasive samples, since they could serve to monitor early responsiveness to the dietary intervention without the need to euthanize the animals and, most important, in human intervention trials. Among accessible samples, blood cells are increasingly being investigated as a source of transcript-based biomarkers in nutritional and obesity studies. It has been shown that gene expression in blood cells can mirror gene expression changes in key tissues in energy homeostasis and respond to both internal and external stimuli (Reynes et al. 2015; Oliver et al. 2013; de Mello et al. 2012).

In a previous study, we used a systematic gene profiling (transcriptomic) approach to evaluate the predictability of short-term, high-fat diet (HFD)-induced changes in adipose tissue gene expression for long-term changes. We identified a set of genes in white adipose tissue (WAT) of mice which are regulated by HFD and whose short-term expression changes (after only 5 days of dietary challenge) are highly predictable for long-term changes (after 12 weeks) (Voigt et al. 2013). We considered this a promising step to establish well-defined, early biomarkers that could shorten animal trials considerably to allow a more rapid and efficient screening of health-promoting food compounds. The current investigation was undertaken with the primary aim to validate this idea. To this end, we conducted short-term (5 day) HFD challenge and intervention trials in mice applying, on top of the HFD, three different dietary interventions which have previously been shown to reduce the development of obesity and associated disorders albeit acting through different physiological mechanisms, i.e., supplementation of epigallocatechin gallate (EGCG) from green tea (Friedrich et al. 2012), replacing part of the dietary lipids by n-3 PUFA (Kus et al. 2011), and a high dietary protein content (Freudenberg et al. 2012). Using quantitative real-time PCR gene expression in epididymal WAT, we identified gene expression of Leptin and Mest [mesoderm-specific transcript (Mest), also known as paternally expressed gene 1 (Peg1)] as early markers of adipose tissue expansion whose HFD-induced expression changes in fat tissue were normalized by the different anti-obesity interventions. Interestingly, in an independent experiment using a different HFD and additional anti-obesity bioactives, we demonstrate similar changes for Mest gene expression in whole blood of mice, thus going one step further in the characterization of Mest gene expression as a biomarker of adipose tissue expansion sensitive to dietary intervention.

\section{Materials and methods}

\section{Animals, diets and interventions}

The experiments were performed in accordance with the national and European ethical guidelines for the use and care of laboratory animals. Experiment 1 was approved by the ethics committee of the Ministry for Environment, Health and Consumer Protection (State Brandenburg, Germany, Permission No. 23-2347-8-2009) and Experiment 2, by the Bioethical Committee of the University of the Balearic Islands (No. 8453, 18-06-2010).

\section{Experiment 1}

Male C57BL/6JRcc mice were obtained from Harlan and Harlan Laboratories $\mathrm{GmbH}$, housed individually at $22{ }^{\circ} \mathrm{C}$ with a 12-h light/dark cycle with ad libitum access to food (standard chow, ssniff Spezialdiäten GmbH, Soest, Germany) and water. At the age of 11-12 weeks, they were divided into 5 different groups matched for body weight $(n=9-10)$ and assigned to one of 5 different semisynthetic diets (Table 1) and fed for 5 days ad libitum. Overall diet composition was based on BIOCLAIMS low-fat diet (LFD) and HFD (Voigt et al. 2013; Hoevenaars et al. 2012). Group 1 was a LFD control group, and group 2 a HFD control group. Both diets have the same components. They are matched in protein energy content and only differ in their carbohydrate/fat content. Groups 3-5 were HFDbased intervention groups. Group 3 (HFD + EGCG) was fed HFD supplemented with $0.5 \%$ of EGCG (TEAVIGO, kindly provided by DSM Nutritional Products Ltd., Basel, Switzerland). TEAVIGO is an extract of green tea (Camellia sinensis), which contains over $94 \%$ of EGCG and $<0.1 \%$ caffeine. Group 4 (HFD + EPAX) received the HFD supplemented with n-3 long-chain polyunsaturated fatty acid concentrate (46\% DHA, $14 \% \mathrm{EPA}$, wt/wt, as triacylglycerols; product EPAX $1050 \mathrm{TG}$; kindly provided by EPAX a.s., Lysaker, Norway) which replaced $15 \% \mathrm{wt} / \mathrm{wt}$ of dietary lipids. In group 5 (HFD/HP), protein content was increased (to 43 energy\%) at the expense of carbohydrates.

Body weight and food intake were recorded daily. After 5 days, mice were killed by cardiac puncture between 8.00 and 12.00 a.m. after isoflurane anesthesia, $2 \mathrm{~h}$ after food withdrawal. Li-heparinized plasma was obtained, and epididymal WAT (eWAT) was snap-frozen in liquid nitrogen and stored at $-80{ }^{\circ} \mathrm{C}$ until analysis. Body composition was determined before the diet switch and at day of dissection using quantitative magnetic resonance (QMR; Bruker's Minispec MQ10, Houston, TX, USA) as described (Klaus et al. 2005; Katterle et al. 2008). 
Table 1 Composition of semisynthetic diets used in Experiment 1

\begin{tabular}{|c|c|c|c|c|c|}
\hline Components & $\begin{array}{l}\text { LFD } \\
\text { (g/kg diet) }\end{array}$ & $\begin{array}{l}\text { HFD } \\
\text { (g/kg diet) }\end{array}$ & $\begin{array}{l}\mathrm{HFD}+\mathrm{EGCG} \\
(\mathrm{g} / \mathrm{kg} \text { diet })\end{array}$ & $\begin{array}{l}\text { HFD + EPAX } \\
\text { (g/kg diet) }\end{array}$ & $\begin{array}{l}\mathrm{HFD} / \mathrm{HP} \\
\text { (g/kg diet) }\end{array}$ \\
\hline Casein & 200 & 267 & 267 & 267 & 500 \\
\hline Wheat starch & 386.5 & 172.5 & 167.5 & 172.5 & - \\
\hline Maltodextrin & 100 & 100 & 100 & 100 & 59.5 \\
\hline Dextrose & 50 & 50 & 50 & 50 & 30 \\
\hline Sucrose & 100 & 100 & 100 & 100 & 100 \\
\hline Cocos oil & 7.74 & 37.8 & 37.8 & 37.8 & 37.8 \\
\hline Sunflower oil & 30.1 & 147 & 147 & 115.5 & 147 \\
\hline Flaxseed & 5.16 & 25.2 & 25.2 & 25.2 & 25.2 \\
\hline EPAX & - & - & - & 31.5 & - \\
\hline Cellulose & 50 & 50 & 50 & 50 & 50 \\
\hline Mineral mixture & 35 & 35 & 35 & 35 & 35 \\
\hline Vitamin mixture & 10 & 10 & 10 & 10 & 10 \\
\hline Choline bitartrate & 2.5 & 2.5 & 2.5 & 2.5 & 2.5 \\
\hline L-Cysteine & 3 & 3 & 3 & 3 & 3 \\
\hline EGCG & - & - & 5 & - & - \\
\hline Total energy $(\mathrm{kJ} / \mathrm{kg})$ & 3865 & 4700 & 4700 & 4700 & 4700 \\
\hline Protein (energy\%) & 23.1 & 23 & 23 & 23 & 42.8 \\
\hline $\mathrm{CHO}$ (energy\%) & 66.9 & 36.8 & 36.8 & 36.8 & 17 \\
\hline Fat (energy\%) & 10.0 & 40.2 & 40.2 & 40.2 & 40.2 \\
\hline
\end{tabular}

$C H O$ carbohydrate, $E G C G$ epigallocatechin gallate, $H F D$ high-fat diet, $L F D$ low-fat diet

\section{Experiment 2}

Four groups of 7-week-old C57BL/6J male mice were used (from CRL España, Barcelona, Spain): a control group fed a defined LFD (D12450J from Research Diets Inc., New Brunswick, NJ, USA) with $10 \%$ energy as fat (LF group), a group fed a defined HFD with $45 \%$ energy as fat (D12451 from Research Diets Inc.) and treated with vehicle (sodium chloride $0.9 \%$ ) (HF group) and groups fed the same HFD and treated with resveratrol $(30 \mathrm{mg} / \mathrm{kg}$ bw/day) or hydroxytyrosol (20 mg/kg bw/day), respectively (HF + RSV and $\mathrm{HF}+\mathrm{HT}$ groups $)(n=10$ animals/group $) .3$-Hydroxyty$\operatorname{rosol}[\geq 98 \%$ (HPLC)] and resveratrol $[\geq 99 \%(\mathrm{GC})]$ were obtained from Sigma (Madrid, Spain). Initial body weight was equal for all animals $(20.1 \pm 0.17 \mathrm{~g}, n=40)$. The animals were housed $2-3$ per cage at $22{ }^{\circ} \mathrm{C}$, with a $12-\mathrm{h}$ light/dark cycle (lights on at 08:00) and free access to food and water. The duration of the experiment was 21 days. Treatments were conducted daily and by oral administration, between 9.00 and 12.00 a.m. Body weight and food intake were regularly monitored. Body composition was analyzed using EchoMRI-700 ${ }^{\mathrm{TM}}$ analyzer (LCC, Houston, TX, USA) after 4 and 20 days of dietary challenge and intervention. Whole blood $(\sim 150 \mu \mathrm{L})$ was taken from facial vein on day 5 and 21 of experiment, at the start of the light cycle, and stored in RNAlater $^{\circledR}$ (Ambion $^{\circledR}$, Life Technologies, Alcobendas, Spain) at $-80{ }^{\circ} \mathrm{C}$. On day 21 , the animals were euthanized under fed conditions within the first $2 \mathrm{~h}$ of the light cycle. WAT depots (epididymal, retroperitoneal and inguinal) were dissected in their entirety and weighed. Inguinal WAT biopsies were fixed with paraformaldehyde for histological examination. Blood collected from the neck was used to measure glucose levels and for the preparation of serum, which was stored at $-80{ }^{\circ} \mathrm{C}$ until analysis.

\section{Circulating parameters}

Random blood glucose was measured before dissection using an automatic device [Accu-Chek Aviva system (Roche Diagnostics)]. Plasma or serum non-esterified fatty acids (NEFA), plasma cholesterol and plasma triglycerides (TG) were measured using colorimetric and enzymatic standard methods (NEFA C kit, Wako Chemicals GmbH, Neuss, Germany; Serum TG Determination Kit, SigmaAldrich, Inc., Steinheim, Germany; Cholesterol liquicolor, Human GmbH, Wiesbaden, Germany, respectively) according to the manufacturer's instructions. Serum insulin levels were measured using commercial ELISA kits [ALPCO Diagnostics, USA (Experiment 1), Mercodia, Uppsala, Sweden (Experiment 2)]. 
Table 2 Biometric data and plasma analyses in animals of Experiment 1

\begin{tabular}{|c|c|c|c|c|c|c|}
\hline & LFD & HFD & HFD/EGCG & HFD/PUFA & $\mathrm{HFD} / \mathrm{HP}$ & ANOVA \\
\hline Initial body weight $(\mathrm{g})$ & $25.4 \pm 0.47$ & $25.3 \pm 0.59$ & $25.2 \pm 0.41$ & $25.3 \pm 0.41$ & $25.7 \pm 0.46$ & ns \\
\hline Final body weight $(\mathrm{g})$ & $26.6 \pm 0.44$ & $27.6 \pm 0.57$ & $26.9 \pm 0.53$ & $27.61 \pm 0.30$ & $26.49 \pm 0.42$ & ns \\
\hline Final fat mass $(\mathrm{g})$ & $6.74 \pm-0.21^{\mathrm{a}}$ & $7.85 \pm 0.29^{c}$ & $7.26 \pm 0.24^{\mathrm{abc}}$ & $7.49 \pm 0.21^{\mathrm{bc}}$ & $6.85 \pm 0.31^{\mathrm{ab}}$ & 0.03 \\
\hline Cumulative energy intake (kJ/animal) & $356 \pm 7.1^{\mathrm{a}}$ & $419 \pm 13^{\mathrm{b}}$ & $362 \pm 14^{\mathrm{a}}$ & $400 \pm 7.4^{\mathrm{ab}}$ & $423 \pm 16^{\mathrm{b}}$ & 0.0002 \\
\hline eWAT weight (\% BW) & $1.81 \pm 0.07$ & $2.04 \pm 0.15$ & $1.70 \pm 0.08$ & $1.85 \pm 0.14$ & $1.67 \pm 0.16$ & ns \\
\hline Liver weight (\% BW) & $4.04 \pm 0.10$ & $3.93 \pm 0.10$ & $3.87 \pm 0.06$ & $4.05 \pm 0.11$ & $4.33 \pm 0.10$ & ns \\
\hline Liver TG (mg/mg protein) & $0.999 \pm 0.164$ & $1.28 \pm 0.281$ & $0.934 \pm 0.203$ & $0.932 \pm 0.162$ & $0.824 \pm 0.167$ & ns \\
\hline Blood glucose $(\mathrm{mmol} / \mathrm{L})$ & $6.36 \pm 0.21$ & $7.23 \pm 0.40$ & $6.80 \pm 0.59$ & $7.16 \pm 0.41$ & $6.51 \pm 0.30$ & ns \\
\hline Plasma insulin $(\mu \mathrm{g} / \mathrm{L})$ & $1.01 \pm 0.14$ & $1.33 \pm 0.17$ & $1.66 \pm 0.37$ & $1.47 \pm 0.25$ & $0.97 \pm 0.09$ & ns \\
\hline Plasma TG (mmol/L) & $0.669 \pm 0.121^{\mathrm{a}}$ & $0.364 \pm 0.044^{\mathrm{b}}$ & $0.330 \pm 0.048^{\mathrm{b}}$ & $0.349 \pm 0.046^{\mathrm{b}}$ & $0.436 \pm 0.040^{\mathrm{ab}}$ & 0.004 \\
\hline Plasma NEFA (mmol/L) & $0.897 \pm 0.045$ & $0.840 \pm 0.063$ & $0.756 \pm 0.084$ & $0.761 \pm 0.052$ & $0.905 \pm 0.065$ & ns \\
\hline Plasma cholesterol (mg/dL) & $140 \pm 4.5^{\mathrm{ab}}$ & $137 \pm 11.1^{\mathrm{ab}}$ & $146 \pm 6.7^{\mathrm{b}}$ & $113 \pm 10.4^{\mathrm{c}}$ & $119 \pm 5.4^{\mathrm{ac}}$ & 0.04 \\
\hline
\end{tabular}

Data are mean \pm SEM $(n=8-10)$. Significant differences between groups were tested by one-way ANOVA and least-significant difference (LSD) post hoc comparisons $(P<0.05)$. Values not sharing a common letter are statistically different

$T G$ triglycerides, $N E F A$ non-esterified fatty acids

\section{RNA extraction and gene expression analysis}

RNA isolation from adipose tissue samples was performed as described before (Keipert et al. 2014). Blood samples stored in RNAlater $^{\circledR}$ were used for total blood RNA extraction using Mouse RiboPure ${ }^{\mathrm{TM}}$-Blood RNA Isolation Kit (Ambion ${ }^{\circledR}$, Life Technologies) and following the manufacturer's instructions. Quantitative real-time PCR was performed essentially as described before (Keipert et al. 2014; Petrov et al. 2015) and followed the MIQE guidelines (Bustin et al. 2009).

\section{Adipose tissue histology}

Specimens of inguinal WAT lobules were fixed by immersion in $4 \%$ paraformaldehyde in $0.1 \mathrm{M}$ phosphate buffer ( $\mathrm{pH}$ 7.4) overnight at $4{ }^{\circ} \mathrm{C}$, dehydrated in a graded series of ethanol, cleared in xylene, and embedded in paraffin blocks. Five-micrometer-thick sections were stained with hematoxylin/eosin to assess tissue morphology using a Zeiss Axioskop 2 microscope equipped with an AxioCam ICc3 digital camera (Carl Zeiss, Barcelona, Spain) as described before (Petrov et al. 2015).

\section{Statistical analysis}

Data are presented as mean \pm SEM. Statistical significance of differences between groups was assessed by oneway ANOVA and least-significant difference (LSD) post hoc comparisons using SPSS 1.9 for windows (SPSS, Chicago, IL, USA) or GraphPad Prism 5 (GraphPad Software, Inc., La Jolla, CA, USA). Threshold of significance was set at $P<0.05$. Values not sharing a common letter are statistically different. Simple correlations with all animals were assessed by Pearson's correlation coefficients using SPSS 1.9 for windows (SPSS, Chicago, IL, USA). Threshold of significance was set at $P<0.05$.

\section{Results}

Leptin and Mest gene expression in adipose tissue as early markers of adipose tissue expansion sensitive to dietary intervention

Obesity-prone mice were challenged with 5 days of feeding either a HFD or one of three versions of the same HFD incorporating anti-obesity ingredients or composition changes, and compared with mice on a LFD. As expected, HFD feeding resulted in an increased overall energy intake. This was attenuated by EGCG supplementation but not by the other treatments (Table 2). Initial and final body weights were not different between the groups. Final fat mass was slightly increased in the HFD and the HFD + PUFA groups compared to the LFD group (Table 2). When the increase (i.e., the difference between day 0 and day 5) was calculated, there was a small but significant increase in both body weight and body fat content in the HFD group compared to LFD (Fig. 1a, b). This was completely inhibited by the high-protein (HFD/ HP) intervention, with HFD + EGCG showing small but non-significant effects and HFD + PUFA showing no effects. Other phenotypic characteristics such as eWAT weight, liver weight and TG content as well as blood glucose, insulin and NEFA content were not affected as expected, due to the brevity of the dietary intervention. 


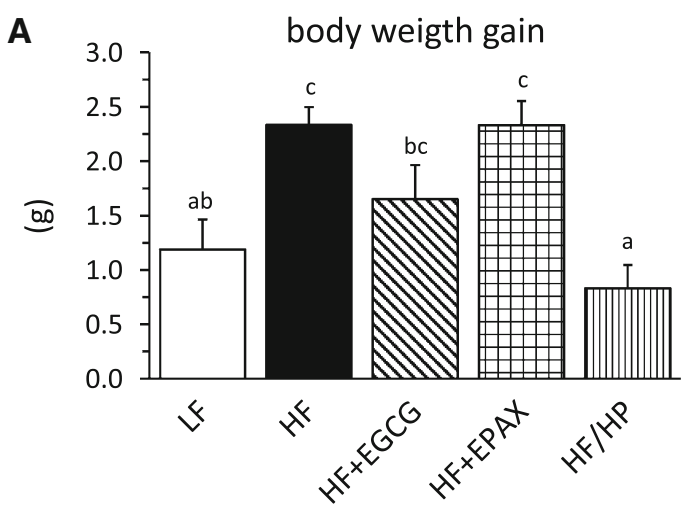

B

body fat gain
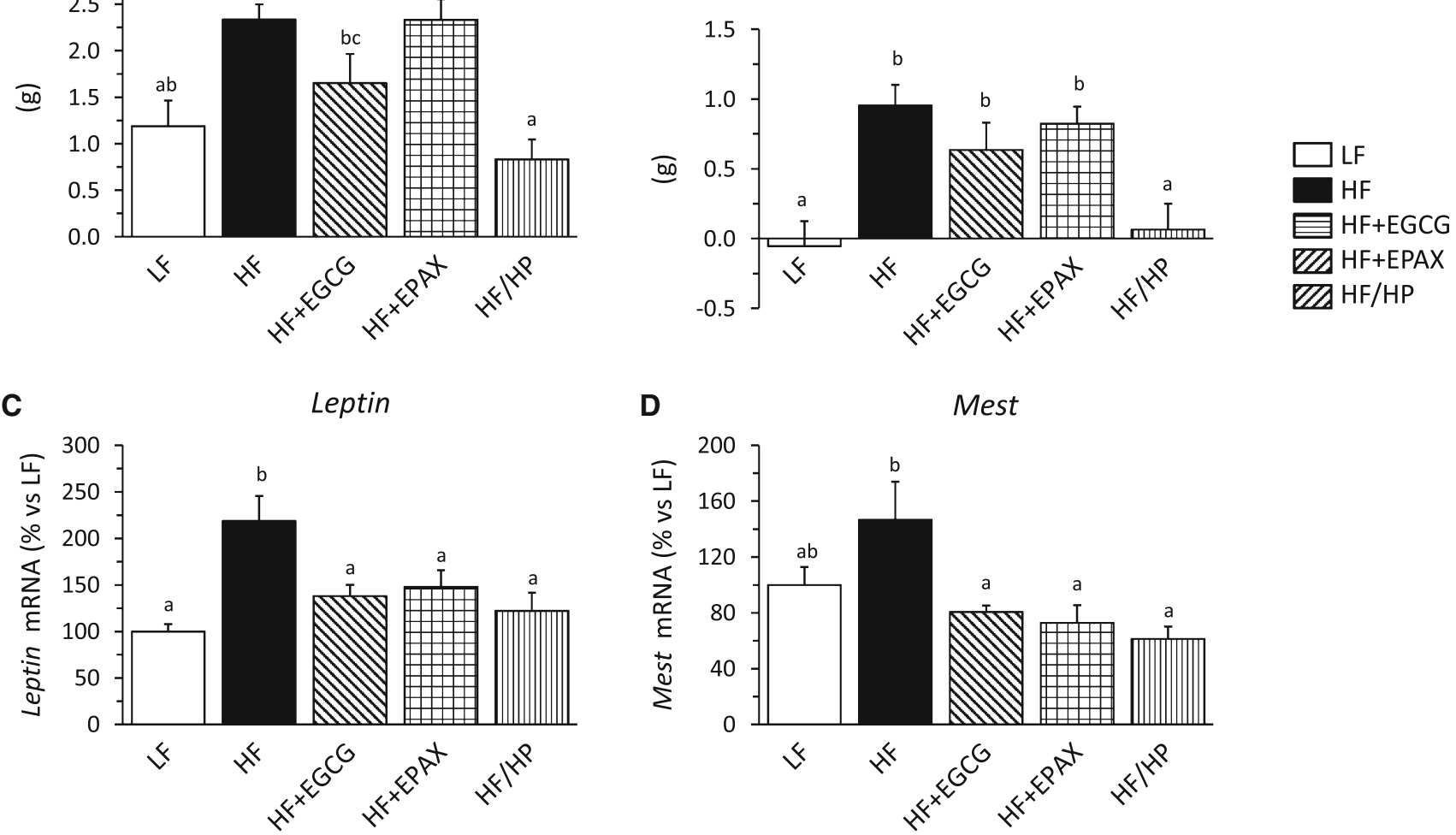

Fig. 1 Body weight gain (a), body fat gain (b) and mRNA expression levels of Leptin (c) and Mest/Pegl (d) in epididymal white adipose tissue (eWAT) of mice fed semi-synthetic low-fat diet (LF), or highfat diet (HF) or a HF diet with different anti-obesity interventions for 5 days. HF + EGCG: supplementation with $0.5 \%$ w/w epigallocatechin gallate (EGCG); HF + EPAX: replacement of $15 \%$ of dietary

lipids by n-3 LC-PUFA (EPAX1050); HF/HP: high-protein diet, partial replacement of carbohydrates by casein to achieve 43 energy $\%$ of protein. Data are means + SEM $(n=7-10)$. Significant differences between groups were tested by one-way ANOVA and leastsignificant difference (LSD) post hoc comparisons $(P<0.05)$. Values not sharing a common letter are statistically different

Interestingly, HFD feeding resulted in a decrease in plasma TG levels which was also seen in the EGCG and PUFA intervention but not in the HP intervention group (Table 2).

Using quantitative RT-PCR, gene expression was analyzed in eWAT, focusing on the genes which were previously identified to show similar regulation after 5 days and 12 weeks of HFD feeding (Voigt et al. 2013). The aim was to identify genes that can serve as robust biomarkers for adipose tissue expansion to be used in short-term intervention trials. Selection criteria were the following: (1) gene expression is affected by 5 -day feeding of a HFD and (2) gene expression is normalized to LFD control values by a dietary anti-obesity intervention. As shown in Table 3, genes of lipid metabolism were mostly downregulated by HFD irrespective of the dietary intervention. Genes of carbohydrate metabolism were affected by HFD and dietary interventions without any consistent pattern. Genes of oxidative phosphorylation were not significantly affected. Genes coding for secreted proteins tended to be upregulated by HFD with minor effects of the interventions.
Interestingly, $R b p 4$ gene expression was downregulated in HFD + EGCG and HFD/HP compared to the HFD group but not by PUFA supplementation.

Finally, of all genes analyzed in eWAT, only 2 fulfilled our criteria as predictive biomarkers for adipose tissue expansion, i.e., Mest/Pegl and the Leptin gene. Leptin gene expression in eWAT was significantly increased after 5 days of HFD (Fig. 1c), even though eWAT weight was not yet significantly increased. All 3 anti-obesity interventions decreased Leptin gene expression similar to levels comparable to LFD control levels. A similar pattern could be seen for Mest gene expression. Although the increase by HFD was not quite significant in the post hoc test, ANOVA showed a highly significant effect $(P=0.0007)$ and all three anti-obesity interventions reduced Mest expression to values even slightly below the LFD control values (Fig. 1d). The only other gene showing a similar expression pattern was Serpine 1 (Table 3) coding for plasminogen activator inhibitor-1, however, without reaching statistical significance. 
Table 3 Gene expression analysis in white adipose tissue (eWAT) of animals in Experiment 1

\begin{tabular}{|c|c|c|c|c|c|c|c|}
\hline Gene name & $\begin{array}{l}\text { Gene } \\
\text { symbol }\end{array}$ & LFD & HFD & HFD/EGCG & HFD/PUFA & $\mathrm{HFD} / \mathrm{HP}$ & ANOVA \\
\hline \multicolumn{8}{|l|}{ Lipid metabolism } \\
\hline ATP citrate lyase & Acly & $1.00 \pm 0.23^{\mathrm{a}}$ & $0.55 \pm 0.03^{\mathrm{a}}$ & $0.42 \pm 0.07^{\mathrm{b}}$ & $0.69 \pm 0.0 .11^{\mathrm{a}}$ & $0.23 \pm 0.23^{\mathrm{b}}$ & 0.002 \\
\hline ELOVL family member 6 & Elovl6 & $1.00 \pm 0.18^{\mathrm{a}}$ & $0.48 \pm 0.10^{\mathrm{b}}$ & $0.33 \pm 0.06^{\mathrm{b}}$ & $0.45 \pm 0.08^{\mathrm{b}}$ & $0.17 \pm 0.03^{\mathrm{b}}$ & $<0.0001$ \\
\hline Fatty acid synthase & Fasn & $1.00 \pm 0.18$ & $0.91 \pm 0.11$ & $0.71 \pm 0.09$ & $0.79 \pm 0.11$ & $0.50 \pm 0.12$ & ns \\
\hline $\begin{array}{l}\text { Acetyl-coenzyme A carboxylase } \\
\text { alpha }\end{array}$ & Acaca & $1.00 \pm 0.13^{\mathrm{a}}$ & $0.44 \pm 0.02^{\mathrm{b}}$ & $0.52 \pm 0.07^{b}$ & $0.62 \pm 0.08^{b}$ & $0.40 \pm 0.05^{\mathrm{b}}$ & $<0.0001$ \\
\hline Malic enzyme 1 & $M e 1$ & $1.00 \pm 0.21^{\mathrm{a}}$ & $0.71 \pm 0.10^{\mathrm{ab}}$ & $0.49 \pm 0.06^{\mathrm{ab}}$ & $0.79 \pm 0.13^{\mathrm{ab}}$ & $0.38 \pm 0.06^{\mathrm{b}}$ & 0.01 \\
\hline $\begin{array}{l}\text { Patatin-like phospholipase } \\
\text { domain-containing protein } 2 \\
\text { (adipose triglyceride lipase) }\end{array}$ & Pnpla2 & $1.00 \pm 0.07^{\mathrm{ab}}$ & $1.14 \pm 0.13^{\mathrm{a}}$ & $0.60 \pm 0.05^{\mathrm{b}}$ & $1.04 \pm 0.10^{\mathrm{a}}$ & $0.88 \pm 0.12^{\mathrm{ab}}$ & 0.004 \\
\hline \multicolumn{8}{|l|}{ Carbohydrate metabolism } \\
\hline Glycogen synthase 2 & Gys2 & $1.00 \pm-0.17^{\mathrm{ab}}$ & $1.19 \pm 0.26^{\mathrm{ab}}$ & $0.70 \pm 0.10^{\mathrm{ab}}$ & $1.67 \pm 0.37^{\mathrm{a}}$ & $0.66 \pm 0.17^{\mathrm{b}}$ & 0.02 \\
\hline $\begin{array}{l}\text { Solute carrier family } 2 \\
\text { (facilitated glucose } \\
\text { transporter), member } 4\end{array}$ & Slc2a4 & $1.00 \pm 0.07^{\mathrm{a}}$ & $1.64 \pm 0.13^{\mathrm{ab}}$ & $1.54 \pm 0.11^{\mathrm{ab}}$ & $0.92 \pm 0.08^{\mathrm{a}}$ & $2.16 \pm 0.37^{\mathrm{b}}$ & 0.0002 \\
\hline Transketolase & $T k t$ & $1.00 \pm 0.12^{\mathrm{a}}$ & $0.58 \pm 0.06^{\mathrm{b}}$ & $0.55 \pm 0.06^{\mathrm{b}}$ & $0.73 \pm 0.10^{\mathrm{ab}}$ & $0.48 \pm 0.06^{\mathrm{b}}$ & 0.0008 \\
\hline \multicolumn{8}{|l|}{ Adipokines } \\
\hline $\begin{array}{l}\text { Serine (or cysteine) peptidase } \\
\text { inhibitor, clade E, member } 1\end{array}$ & Serpine1 & $1.00 \pm 0.22$ & $1.36 \pm 0.25$ & $0.82 \pm 0.14$ & $0.79 \pm 0.14$ & $0.69 \pm 0.14$ & $\mathrm{~ns}$ \\
\hline Haptoglobin & $H p$ & $1.00 \pm 0.13^{\mathrm{a}}$ & $1.22 \pm 0.16^{\mathrm{ab}}$ & $1.23 \pm 0.08^{\mathrm{ab}}$ & $1.03 \pm 0.08^{\mathrm{a}}$ & $1.66 \pm 0.21^{\mathrm{b}}$ & 0.02 \\
\hline $\begin{array}{l}\text { Retinol-binding protein } 4 \text {, } \\
\text { plasma }\end{array}$ & Rbp4 & $1.00 \pm 0.14^{\mathrm{ab}}$ & $1.45 \pm 0.26^{\mathrm{a}}$ & $0.49 \pm 0.05^{\mathrm{b}}$ & $1.42 \pm 0.17^{\mathrm{a}}$ & $0.55 \pm 0.05^{\mathrm{b}}$ & $<0.0001$ \\
\hline \multicolumn{8}{|l|}{ Oxidative phosphorylation } \\
\hline $\begin{array}{l}\text { NADH dehydrogenase } \\
\quad \text { (ubiquinone) Fe-S protein } 1\end{array}$ & Ndufs 1 & $1.00 \pm 0.06$ & $0.97 \pm 0.05$ & $0.92 \pm 0.05$ & $1.04 \pm 0.08$ & $0.95 \pm 0.05$ & ns \\
\hline $\begin{array}{l}\text { ATP synthase, } \mathrm{H}+\text { transporting, } \\
\text { mitochondrial F1 complex, } \\
\text { gamma polypeptide } 1\end{array}$ & Atp 5c1 & $1.00 \pm 0.05$ & $1.17 \pm 0.06$ & $0.95 \pm 0.04$ & $0.99 \pm 0.08$ & $0.99 \pm 0.06$ & ns \\
\hline \multicolumn{8}{|l|}{ Miscellaneous } \\
\hline Epithelial membrane protein 1 & Empl & $1.00 \pm 0.06$ & $1.09 \pm 0.14$ & $1.37 \pm 0.18$ & $0.92 \pm 0.14$ & $1.17 \pm 0.16$ & ns \\
\hline $\begin{array}{l}\text { Transformation-related protein } \\
53 \text {-inducible nuclear protein } 2\end{array}$ & Trp53inp2 & $1.00 \pm 0.07$ & $1.57 \pm 0.13$ & $1.41 \pm 0.15$ & $1.27 \pm 0.15$ & $1.25 \pm 0.14$ & ns \\
\hline $\begin{array}{l}\text { Thyroid hormone responsive } \\
\text { SPOT14 homolog (rattus) }\end{array}$ & Thrsp & $1.00 \pm 0.12$ & $1.00 \pm 0.10$ & $0.86 \pm 0.13$ & $1.36 \pm 0.20$ & $1.32 \pm 0.17$ & ns \\
\hline $\begin{array}{l}\text { Protein phosphatase } 1 \text {, } \\
\text { regulatory (inhibitor) subunit } \\
1 \mathrm{~A}\end{array}$ & Ppplrla & $1.00 \pm 0.07$ & $0.98 \pm 0.11$ & $0.93 \pm 0.07$ & $1.32 \pm 0.16$ & $0.94 \pm 0.11$ & ns \\
\hline $\begin{array}{l}\text { Cell death-inducing DNA } \\
\text { fragmentation factor, alpha } \\
\text { subunit-like effector A }\end{array}$ & Cidea & $1.00 \pm 0.13^{\mathrm{a}}$ & $0.52 \pm 0.09^{\mathrm{b}}$ & $0.59 \pm 0.06^{\mathrm{b}}$ & $0.64 \pm 0.09^{\mathrm{ab}}$ & $0.45 \pm 0.05^{\mathrm{b}}$ & 0.001 \\
\hline Insulin-induced gene 1 & Insig1 & $1.00 \pm 0.15^{\mathrm{a}}$ & $1.02 \pm 0.10^{\mathrm{a}}$ & $0.67 \pm 0.10^{\mathrm{ab}}$ & $1.00 \pm 0.15^{\mathrm{a}}$ & $0.42 \pm 0.08^{\mathrm{b}}$ & 0.003 \\
\hline
\end{tabular}

Data are mean \pm SEM $(n=7-8)$. Significant differences between groups were tested by one-way ANOVA and least-significant difference (LSD) post hoc comparisons $(P<0.05)$. Values not sharing a common letter are statistically different

\section{Mest gene expression in whole blood as an early marker of adipose tissue expansion sensitive to dietary intervention}

Obesity-prone mice were challenged for 21 days with a HFD without or with simultaneous daily oral treatment with putative anti-obesity bioactives (hydroxytyrosol or resveratrol), and compared with mice on a LFD for parameters including Leptin and Mest expression in whole blood after 5 days of HFD feeding. There were no significant differences between groups in cumulative energy intake over the 21-day experimental period (not shown). Although final body weight was not affected by 21 days of HFD (not shown), body weight gain was greater in the HFD-fed groups 
compared with the LF group. This was evident already after only 3 days on the HFD (Fig. 2a), as expected from previous results [(Voigt et al. 2013) and current Experiment 1]. Hydroxytyrosol-treated mice showed a trend to reduced body weight gain on the HFD that reached statistical significance between days 15 and 18. After 4 days, animals on the HFD treated with vehicle already had increased percent body fat content compared with the LF group, whereas the difference did not reach statistical significance for the animals on the HFD treated with either of the two bioactives (Fig. 2b). Body fat content at day 20 (Fig. 2c), adiposity index (defined as the sum of the mass of all WAT depots dissected expressed as percentage of body weight) at day 21 (not shown) and subcutaneous (inguinal) WAT depot mass at day 21 (Fig. 2d) were similarly increased in the three HFD-fed groups relative to the LF group. However, the accrual of visceral fat (epididymal and retroperitoneal depots) was decreased in the hydroxytyrosol- and, to a lesser extent, the resveratrol-treated mice (Fig. 2e, f), which therefore displayed a higher subcutaneous/visceral fat ratio at the end of the experiment compared with the vehicle-treated HF group (Fig. 2g). Moreover, although subcutaneous (inguinal) WAT depot mass was similarly increased by HFD irrespective of bioactive treatment, hydroxytyrosol- and resveratrol-treated mice displayed smaller inguinal WAT adipocytes at the end of the experiment, as evidenced by morphological examination (Fig. 3). Collectively, these results indicate that the bioactives tested, especially hydroxytyrosol, beneficially impacted on body adiposity, counteracting the effects of the obesogenic diet. Additionally, the increase in blood glucose levels following 21 days of HFD was ameliorated by bioactive treatment, especially hydroxytyrosol (Fig. 2h), while serum insulin and NEFA levels showed no significant differences between groups (Fig. 2i, j).
A

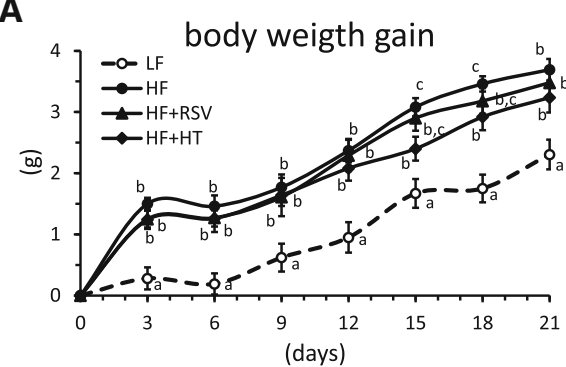

B

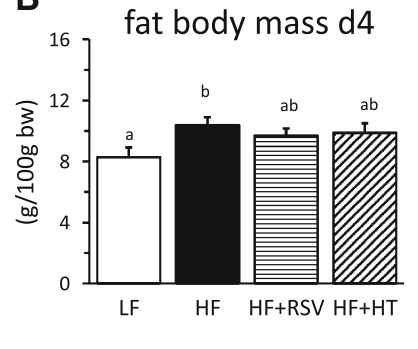

C

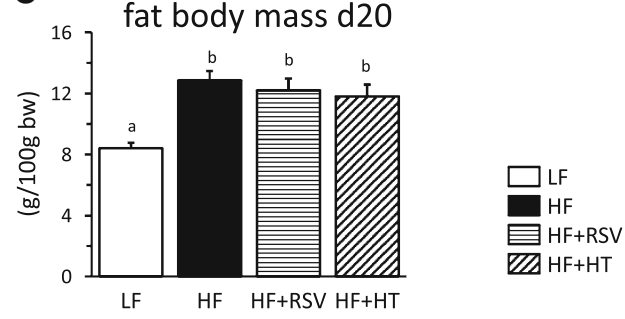

D

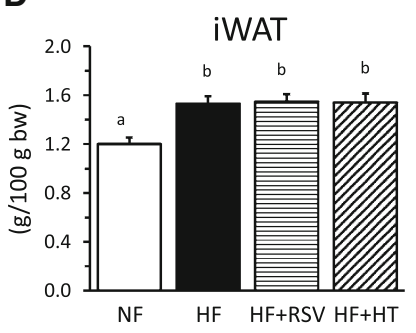

E

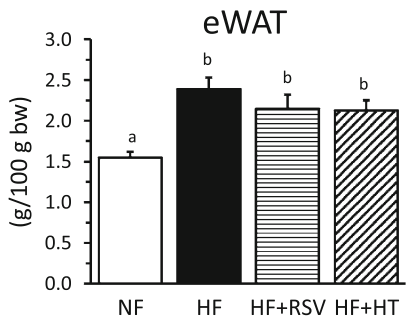

I
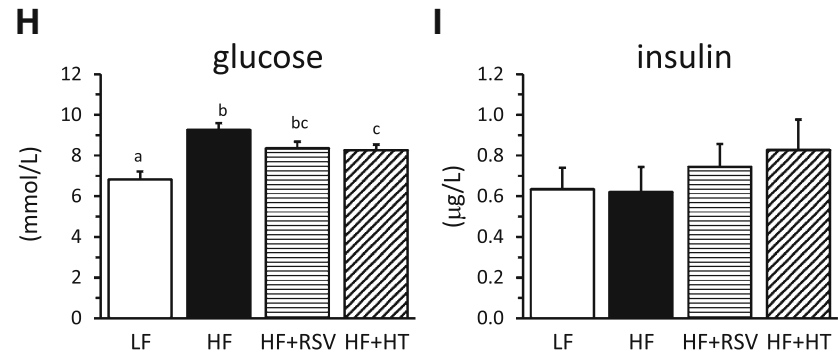

Fig. 2 Biometrics and serum parameters in obesity-prone mice fed during 21 days a semi-synthetic low-fat diet (LF group), a high-fat diet (HF group) or a high-fat diet with simultaneous oral daily treatment with resveratrol $(30 \mathrm{mg} / \mathrm{kg}$ bw/day, $\mathrm{HF}+\mathrm{RSV}$ group $)$ or hydroxytyrosol $(20 \mathrm{mg} / \mathrm{kg}$ bw/day, HF $+\mathrm{HT}$ group). The panels show development of body weight gain (a), fat body content at day 4 (b) and 20 (c) and the following parameters measured at the final end point of the experiment (day 21): inguinal (d), epididymal (e) and
$\mathbf{F}$

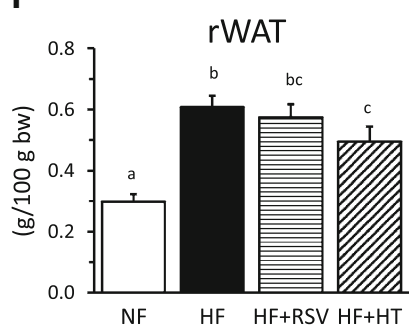

G

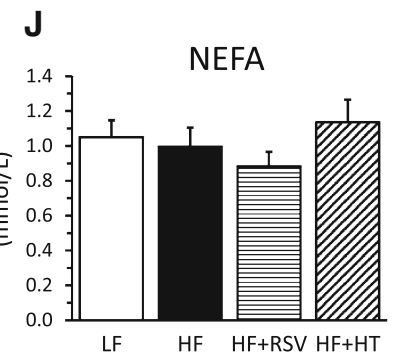

retroperitoneal (f) white adipose tissue depot weights, subcutaneous/ visceral fat ratio (g) and serum glucose (h), insulin (i) and nonesterified fatty acids (NEFA, j) levels. Data are means + SEM $(n=7-10)$. Significant differences between groups were tested by one-way ANOVA and least-significant difference (LSD) post hoc comparisons $(P<0.05)$. Values not sharing a common letter are statistically different 
Fig. 3 Representative optical images of sections of inguinal white adipose tissue in animals in the LF group (a), the HF group (b), the HF + RSV group (c) and the HF + HT group (d) described in the legend to Fig. 2 following 21 days of dietary intervention, illustrating differences in adipocyte size (the scale bar corresponds to $100 \mu \mathrm{m})$
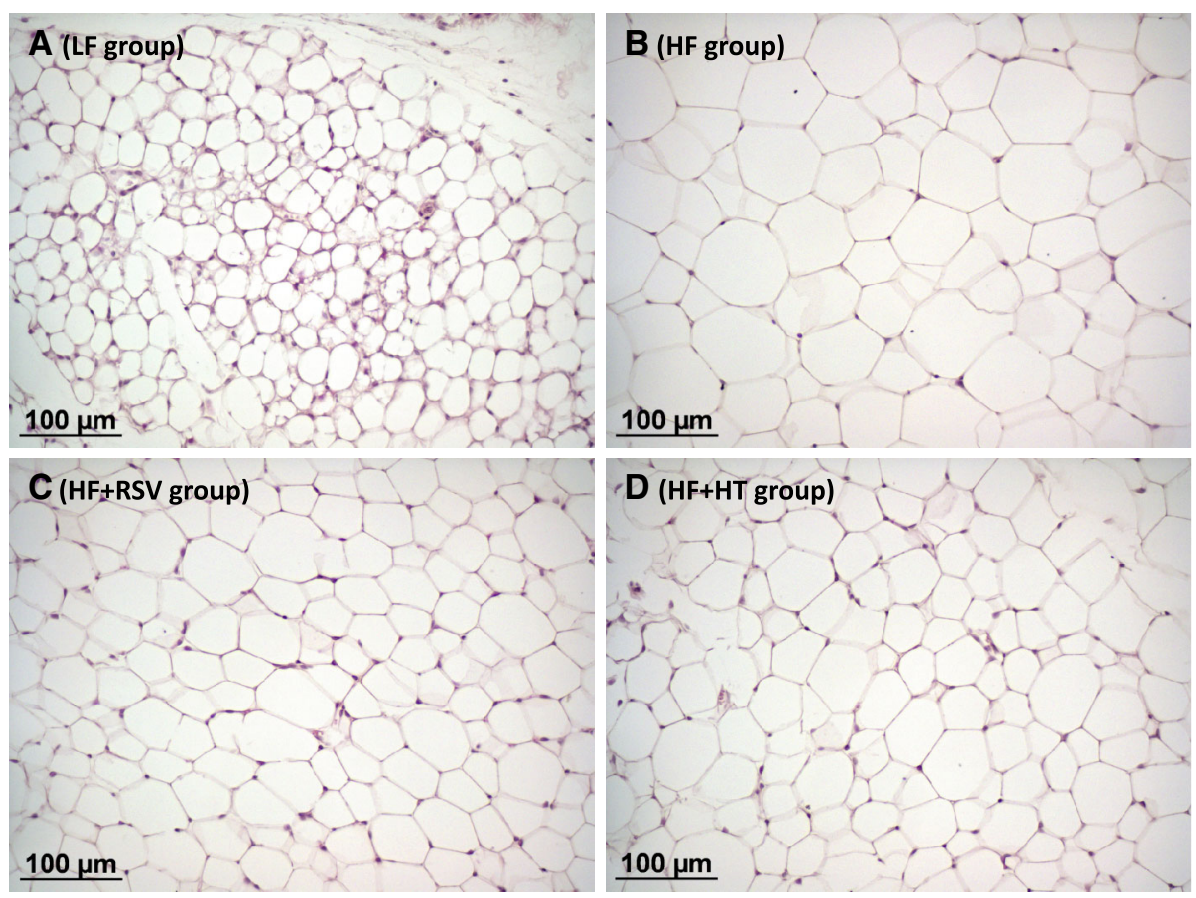

Gene expression analysis of Mest in whole blood revealed a considerable $(\sim 80 \%)$ acute increase after 5 days on the HFD that was markedly attenuated after a more prolonged challenge of 21 days (Fig. 4a, b). The acute increase of Mest expression in whole blood upon HFD feeding was suppressed by simultaneous treatment with hydroxytyrosol, but not with resveratrol (Fig. 4a). Additionally, mRNA levels of Mest in whole blood after 21 days on HFD tended to be lower in the two groups of mice fed the HFD and treated with bioactive compared with the HFD group that received the vehicle (Fig. 4b). Remarkably, when all animals in Experiment 2 were included, Mest expression in total blood at day 5 positively correlated with adiposity and negatively with lean body mass and the subcutaneous/visceral fat ratio at the end of the experiment (Fig. 4c-e). In fact, Mest expression in total blood at day 5 positively correlated with the mass of all WAT depots at the end of the experiment and the positive correlation was maintained for Mest expression in total blood at day 21 and eWAT mass (not shown). Leptin mRNA was barely detectable in whole blood by Q-PCR, because in most samples analyzed $\mathrm{Ct}$ (cycle threshold) values were above 35 and thus close to or below detection threshold.

\section{Discussion}

Predictive biomarkers of WAT accretion are of interest since they can serve to shorten animal trials assaying novel ingredients or other dietary interventions for anti-obesity activity and, particularly if linked to easily accessible noninvasive samples, to proof effects and identify responders in human intervention trials of this type. They may, therefore, find applicability in the substantiation of health claims made on food regarding obesity, adiposity and related health problems. In the current work, we sought to derive such biomarkers starting from previous gene expression profiling data of WAT after short- and longterm HFD feeding in mice (Voigt et al. 2013). First, dietary anti-obesity intervention strategies on top of a HFD were used to identify short-term gene expression changes in mouse WAT that were both predictive of long-term changes and sensitive to dietary interventions. Second, genes identified were assessed in whole blood in an independent experiment using additional anti-obesity bioactives. Our results suggest that Mest expression in WAT and whole blood could be used as a predictive biomarker for fat mass expansion.

Altogether, five different anti-obesity interventions have been used here which have been previously shown to reduce the development of obesity and associated disorders albeit acting through different physiological mechanisms. Dietary supplementation of EGCG, the green tea bioactive polyphenol, prevented the development of HFD-induced obesity in a dose-dependent manner in mice after 4 weeks (Klaus et al. 2005). This was shown to be due to decreased intestinal fat absorption and transport (i.e., higher energy excretion) and increased oxidation of dietary lipids linked to a reduced incorporation of dietary lipids into various tissues (Friedrich et al. 2012). Another intervention was the 
A
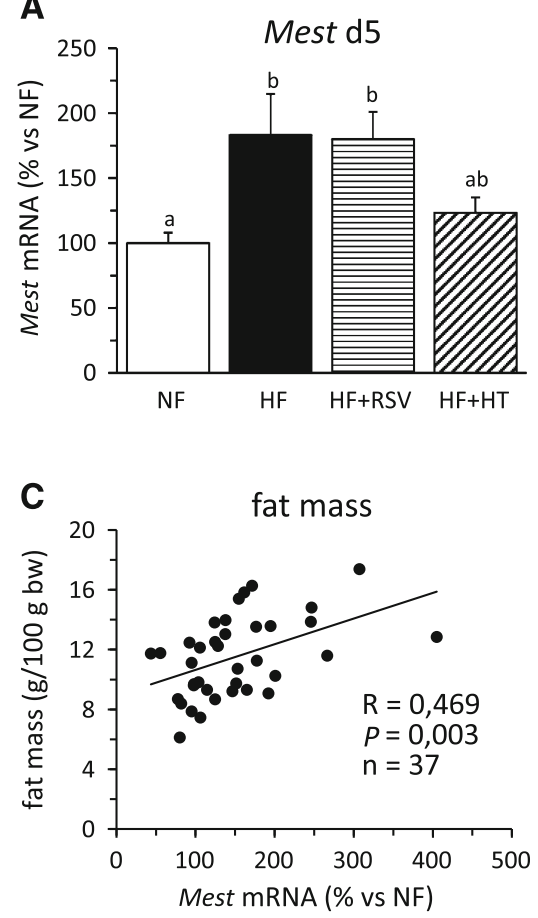

B
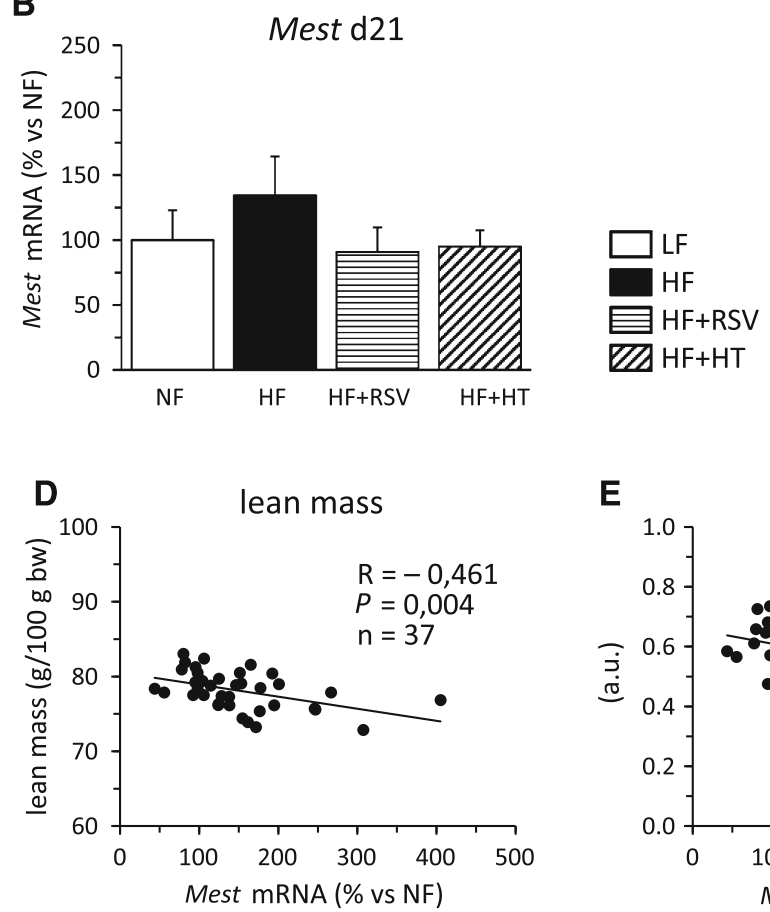

E

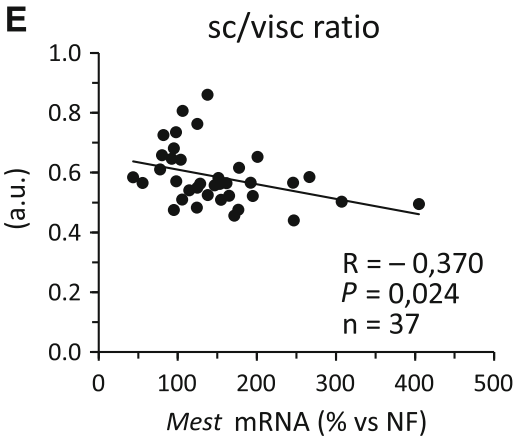

Fig. 4 mRNA expression levels of Mest in whole blood at day 5 (a) and 21 (b) and correlation of whole blood Mest mRNA levels at day 5 with body fat mass (c) and lean body mass (d) at day 20, and with subcutaneous/visceral fat ratio (e) at the end of the experiment (day 21) in groups of animals as defined in the legend to Fig. 2. Gene expression data are means + SEM $(n=8-10)$. Significant

replacement of $15 \%$ of dietary lipids with EPAX-1050, a triglyceride mixture containing $60 \%$ of n-3 LC-PUFAs from marine origin. The experimental setup was the same which has been shown to reduce feed efficiency, fat depots and liver TG content, and to increase insulin sensitivity after 8 weeks of HFD feeding in mice (Kus et al. 2011) linked to an increased metabolic flexibility of skeletal muscle, with n-3 LC-PUFA supporting complete oxidation of fatty acids in mitochondria (Horakova et al. 2012). A third intervention was an increase in the protein content of the HFD to over $40 \%$. This has been shown to result in decreased body weight, body fat and hepatic TG accumulation, as well as increased insulin sensitivity compared to a HFD with adequate protein content after 20 weeks of feeding (Freudenberg et al. 2012). It was suggested that the positive effects of high-protein diets on metabolic syndrome associated traits are acutely due to satiety effects possibly linked to amino nitrogen intake and the subsequent suppression of liver lipogenesis (Freudenberg et al. 2013). Finally, hydroxytyrosol and resveratrol are two phenolic phytochemicals abundant in extra-virgin olive oil and grapes and red wine, respectively. For these two compounds, beneficial effects on metabolic health have been demonstrated, including an anti-obesity action in differences between groups were tested by one-way ANOVA and least-significant difference (LSD) post hoc comparisons $(P<0.05)$. Values not sharing a common letter are statistically different. The correlation analyses were performed with all animals. Pearson's correlation indexes, statistical significance and $n$ are indicated

animals (Cao et al. 2014; Aguirre et al. 2014) which has been related to interactions with adipogenesis and adipocyte lipid and energy metabolism (Hao et al. 2010; Drira and Sakamoto 2014; Mercader et al. 2011; Gomez-Zorita et al. 2013) among other mechanisms. The dietary interventions chosen thus represent a broad range of treatments shown to attenuate or prevent the development of HFDinduced obesity and associated features of the metabolic syndrome in different studies.

Studies indicate that WAT rapidly responds to changes in the nutritional status with changes in gene expression (Voigt et al. 2013). Therefore, we first sought to identify genes whose modulations in WAT by 5 days of HFD feeding were similarly counteracted by three different antiobesity intervention treatments (EGCG from green tea, n-3 PUFA and high dietary protein content). Using gene expression profiling, we have previously shown that about $80 \%$ of all genes differentially expressed in eWAT after 5 days of HFD feeding were downregulated. These downregulated genes were mainly related to metabolic pathways such as lipogenesis, carbohydrate metabolism and mitochondrial function (Voigt et al. 2013). Our current analysis showed that none of these genes fulfilled our criteria for robust biomarkers. Although their downregulation 
by HFD could mainly be confirmed, their expression levels were not normalized by the anti-obesity interventions suggesting that genes crucial for lipid and glucose metabolism are not suitable as robust early biomarkers for adipose tissue health and expansion. Much less genes were upregulated by HFD such as genes coding for secreted proteins including leptin, serpine 1 , haptoglobin and rbp4. Of these genes, only Leptin and Mest/Pegl gene expression was consistently normalized by the different anti-obesity interventions. Thus, we show that gene expression of both Leptin and Mest was not only increased by short-term HFD feeding, but more importantly that their expression levels were completely normalized to LFD control levels in the HFD groups with simultaneous anti-obesity treatments.

Since its first discovery more than 20 years ago (Zhang et al. 1994), leptin has been well established as an adipose tissue-derived adipokine reflecting adipose tissue mass (Friedman 2014). It is long known that Leptin gene expression is correlated with adipocyte lipid content (Maffei et al. 1995), but surprisingly, the exact molecular mechanisms regulating its gene expression and secretion are still unknown today. Our data suggest that Leptin gene expression might be acutely down- or upregulated by catabolic or anabolic state of adipocytes, respectively.

Mest has also been known for about 20 years when it was discovered as a mesodermal imprinted, paternally expressed gene (Kaneko-Ishino et al. 1995) important for embryonic development (Lefebvre et al. 1998). Later it was shown to be highly expressed in adipose tissue, and its expression was linked to adipocyte differentiation (Kadota et al. 2012; Takahashi et al. 2005). Although the exact biochemical function of the Mest product is still unknown, it is suggested to play a role in adipocyte differentiation by inhibition of the Wnt signaling pathway (Jung et al. 2011). Several lines of evidence point to Mest as an acute marker of adipose tissue and fat cell expansion: Mest gene expression was highly increased in WAT of obese mice in diet-induced obesity as well as in genetic obesity (Takahashi et al. 2005; Okada et al. 2009; Moraes et al. 2003; Jura et al. 2015) which was linked to a positive energy balance during the most rapid phase of fat mass expansion (Nikonova et al. 2008; Kozak et al. 2010). Across several mouse strains, Mest expression in WAT was found to be associated with body weight (Anunciado-Koza et al. 2015). It has previously been shown that Mest expression in WAT was significantly increased already after 2 days of HFD feeding (Nikonova et al. 2008). In rats, dietary treatments during the suckling period that differentially affected inguinal adipocyte size at weaning also affected Mest gene expression of the same depot similarly (Musinovic et al. 2014). Differences in Mest gene expression observed in mouse WAT biopsies at an early age correlated with adiposity after 8 weeks of HFD feeding in mice (Koza et al.
2006). Furthermore, overexpression of Mest in 3T3-L1 adipocytes and transgenic mice led to an increased expression of adipocyte gene markers and considerably enlarged adipocytes (Takahashi et al. 2005). Together, this suggests that Mest is not only a biomarker for fat mass expansion but also causally linked to adipose expansion. In humans, evidence has been provided that epigenetic malprogramming of MEST may contribute to obesity predisposition throughout life, and of decreased blood MEST methylation in adults with morbid obesity compared with normal-weight controls (El Hajj et al. 2013).

In the first approach, we identified Leptin and Mest gene expression in WAT as early, predictive biomarkers of WAT accretion. In the second approach, we aimed to assess the translatability of these findings to whole bloodbased expression data. The level of expression of Leptin gene in mouse blood is defined "low" by the web-based software anatomy tool of Genevestigator V3 (https://gene vestigator.com/gv/index.jsp) (Hruz et al. 2008), and expression of the Leptin gene was found to be undetectable in rat peripheral blood mononuclear cells (Reynés B, Oliver $\mathrm{P}$ and Palou $\mathrm{A}$, personal communication). In line with these observations, only Mest gene expression could be consistently detected in whole blood of our mice. Our results are first to reveal an induction of Mest gene expression in whole blood following the intake of an obesogenic diet. Remarkably, this was especially strong in the short term (day 5) although still evident after a more prolonged HFD challenge (21 days). Importantly, this response was suppressed or attenuated by simultaneous treatment with anti-obesity bioactives, hydroxytyrosol and, to a lesser extent, resveratrol. Importantly, treatment with these bioactives, particularly hydroxytyrosol, ameliorated other HFD-induced changes in our model system, such as increased blood glucose levels, visceral WAT gain and subcutaneous adipocyte size, thus favouring a healthier phenotype and adipose organ and validating their use as anti-obesity agents in our design. Most remarkably, Mest expression in total blood at day 5 correlated with measures of body composition at day 20-21, in a sense that greater Mest expression was associated with increased adiposity, decreased percent lean body mass and relatively more visceral fat (decreased subcutaneous/visceral fat ratio). To our knowledge, these results are first to show the potential of Mest gene expression in whole blood as an early, predictive marker of WAT expansion.

\section{Conclusions}

Leptin and Mest gene expression in eWAT and Mest gene expression in whole blood were upregulated by short-term (5 days) HFD feeding and normalized by different dietary 
interventions previously shown to have anti-obesity effects in the long-term. Leptin and Mest gene expression in fat depots as well as Mest gene expression in whole blood can thus be considered as predictive markers of adipose tissue accretion which could be useful for the rapid screening and evaluation of bioactive compounds/interventions with potential anti-obesity action.

Acknowledgments The research leading to these results has received funding from the European Union's Seventh Framework Program FP7 2007-2013 under Grant Agreement No. 244995 (BIOCLAIMS Project). The authors thank Mario Rodríguez-Baeza for his help in experiment 2 during his Master studies. Excellent technical assistance provided by Antje Sylvester, Carolin Borchert and Enzo Ceresi is acknowledged.

\section{Compliance with Ethical Standards}

Conflict of interest None.

\section{References}

Aguirre L, Fernandez-Quintela A, Arias N, Portillo MP (2014) Resveratrol: anti-obesity mechanisms of action. Molecules 19:18632-18655

Anunciado-Koza RP, Higgins DC, Koza RA (2015) Adipose tissue Mest and Sfrp5 are concomitant with variations of adiposity among inbred mouse strains fed a non-obesogenic diet. Biochimie. doi:10.1016/j.biochi.2015.05.007

Bustin SA, Benes V, Garson JA, Hellemans J, Huggett J, Kubista M, Mueller R, Nolan T, Pfaffl MW, Shipley GL, Vandesompele J, Wittwer CT (2009) The MIQE guidelines: minimum information for publication of quantitative real-time PCR experiments. Clin Chem 55:611-622

Cao K, Xu J, Zou X, Li Y, Chen C, Zheng A, Li H, Szeto IM, Shi Y, Long J, Liu J, Feng Z (2014) Hydroxytyrosol prevents dietinduced metabolic syndrome and attenuates mitochondrial abnormalities in obese mice. Free Radic Biol Med 67:396-407

de Mello VD, Kolehmanien M, Schwab U, Pulkkinen L, Uusitupa M (2012) Gene expression of peripheral blood mononuclear cells as a tool in dietary intervention studies: what do we know so far? Mol Nutr Food Res 56:1160-1172

Drira R, Sakamoto K (2014) Hydroxytyrosol stimulates lipolysis via A-kinase and extracellular signal-regulated kinase activation in 3T3-L1 adipocytes. Eur J Nutr 53:743-750

El Hajj N, Pliushch G, Schneider E, Dittrich M, Muller T, Korenkov M, Aretz M, Zechner U, Lehnen H, Haaf T (2013) Metabolic programming of MEST DNA methylation by intrauterine exposure to gestational diabetes mellitus. Diabetes 62:1320-1328

Freudenberg A, Petzke KJ, Klaus S (2012) Comparison of highprotein diets and leucine supplementation in the prevention of metabolic syndrome and related disorders in mice. J Nutr Biochem 23:1524-1530

Freudenberg A, Petzke KJ, Klaus S (2013) Dietary L-leucine and Lalanine supplementation have similar acute effects in the prevention of high-fat diet-induced obesity. Amino Acids 44:519-528

Friedman J (2014) 20 years of leptin: leptin at 20: an overview. J Endocrinol 223:T1-T8

Friedrich M, Petzke KJ, Raederstorff D, Wolfram S, Klaus S (2012) Acute effects of epigallocatechin gallate from green tea on oxidation and tissue incorporation of dietary lipids in mice fed a high-fat diet. Int J Obes (Lond) 36:735-743

Gomez-Zorita S, Treguer K, Mercader J, Carpene C (2013) Resveratrol directly affects in vitro lipolysis and glucose transport in human fat cells. J Physiol Biochem 69:585-593

Hao J, Shen W, Yu G, Jia H, Li X, Feng Z, Wang Y, Weber P, Wertz K, Sharman E, Liu J (2010) Hydroxytyrosol promotes mitochondrial biogenesis and mitochondrial function in 3T3-L1 adipocytes. J Nutr Biochem 21:634-644

Hoevenaars FP, van Schothorst EM, Horakova O, Voigt A, Rossmeisl M, Pico C, Caimari A, Kopecky J, Klaus S, Keijer J (2012) BIOCLAIMS standard diet (BIOsd): a reference diet for nutritional physiology. Genes Nutr 7:399-404

Horakova O, Medrikova D, van Schothorst EM, Bunschoten A, Flachs P, Kus V, Kuda O, Bardova K, Janovska P, Hensler M, Rossmeisl M, Wang-Sattler R, Prehn C, Adamski J, Illig T, Keijer J, Kopecky J (2012) Preservation of metabolic flexibility in skeletal muscle by a combined use of n-3 PUFA and rosiglitazone in dietary obese mice. PLoS One 7:e43764

Hruz T, Laule O, Szabo G, Wessendorp F, Bleuler S, Oertle L, Widmayer P, Gruissem W, Zimmermann P (2008) Genevestigator v3: a reference expression database for the meta-analysis of transcriptomes. Adv Bioinform 2008:420747

Jung H, Lee SK, Jho EH (2011) Mest/Peg1 inhibits Wnt signalling through regulation of LRP6 glycosylation. Biochem J 436:263-269

Jura M, Jaroslawska J, Chu DT, Kozak LP (2015) Mest and Sfrp5 are biomarkers for healthy adipose tissue. Biochimie. doi:10.1016/j. biochi.2015.05.006

Kadota Y, Yanagawa M, Nakaya T, Kawakami T, Sato M, Suzuki S (2012) Gene expression of mesoderm-specific transcript is upregulated as preadipocytes differentiate to adipocytes in vitro. J Physiol Sci 62:403-411

Kaneko-Ishino T, Kuroiwa Y, Miyoshi N, Kohda T, Suzuki R, Yokoyama M, Viville S, Barton SC, Ishino F, Surani MA (1995) Peg1/Mest imprinted gene on chromosome 6 identified by cDNA subtraction hybridization. Nat Genet 11:52-59

Katterle Y, Keipert S, Hof J, Klaus S (2008) Dissociation of obesity and insulin resistance in transgenic mice with skeletal muscle expression of uncoupling protein 1. Physiol Genomics 32:352-359

Keipert S, Ost M, Johann K, Imber F, Jastroch M, van Schothorst EM, Keijer J, Klaus S (2014) Skeletal muscle mitochondrial uncoupling drives endocrine cross-talk through the induction of FGF21 as a myokine. Am J Physiol Endocrinol Metab 306:E469-E482

Klaus S, Rudolph B, Dohrmann C, Wehr R (2005a) Expression of uncoupling protein 1 in skeletal muscle decreases muscle energy efficiency and affects thermoregulation and substrate oxidation. Physiol Genomics 21:193-200

Klaus S, Pultz S, Thone-Reineke C, Wolfram S (2005b) Epigallocatechin gallate attenuates diet-induced obesity in mice by decreasing energy absorption and increasing fat oxidation. Int $\mathrm{J}$ Obes (Lond) 29:615-623

Koza RA, Nikonova L, Hogan J, Rim JS, Mendoza T, Faulk C, Skaf J, Kozak LP (2006) Changes in gene expression foreshadow dietinduced obesity in genetically identical mice. PLoS Genet 2:e81

Kozak LP, Newman S, Chao PM, Mendoza T, Koza RA (2010) The early nutritional environment of mice determines the capacity for adipose tissue expansion by modulating genes of caveolae structure. PLoS One 5:e11015

Kus V, Flachs P, Kuda O, Bardova K, Janovska P, Svobodova M, Jilkova ZM, Rossmeisl M, Wang-Sattler R, Yu Z, Illig T, Kopecky J (2011) Unmasking differential effects of rosiglitazone and pioglitazone in the combination treatment with $n-3$ fatty acids in mice fed a high-fat diet. PLoS One 6:e27126 
Lefebvre L, Viville S, Barton SC, Ishino F, Keverne EB, Surani MA (1998) Abnormal maternal behaviour and growth retardation associated with loss of the imprinted gene Mest. Nat Genet 20:163-169

Maffei M, Halaas J, Ravussin E, Pratley RE, Lee GH, Zhang Y, Fei H, Kim S, Lallone R, Ranganathan S et al (1995) Leptin levels in human and rodent: measurement of plasma leptin and ob RNA in obese and weight-reduced subjects. Nat Med 1:1155-1161

Mercader J, Palou A, Bonet ML (2011) Resveratrol enhances fatty acid oxidation capacity and reduces resistin and retinol-binding protein 4 expression in white adipocytes. J Nutr Biochem $22: 828-834$

Moraes RC, Blondet A, Birkenkamp-Demtroeder K, Tirard J, Orntoft TF, Gertler A, Durand P, Naville D, Begeot M (2003) Study of the alteration of gene expression in adipose tissue of dietinduced obese mice by microarray and reverse transcriptionpolymerase chain reaction analyses. Endocrinology 144:4773-4782

Musinovic H, Bonet ML, Granados N, Amengual J, von Lintig J, Ribot J, Palou A (2014) Beta-carotene during the suckling period is absorbed intact and induces retinoic acid dependent responses similar to preformed vitamin A in intestine and liver, but not adipose tissue of young rats. Mol Nutr Food Res 58:2157-2165

Nikonova L, Koza RA, Mendoza T, Chao PM, Curley JP, Kozak LP (2008) Mesoderm-specific transcript is associated with fat mass expansion in response to a positive energy balance. FASEB $\mathrm{J}$ 22:3925-3937
Okada Y, Sakaue H, Nagare T, Kasuga M (2009) Diet-induced upregulation of gene expression in adipocytes without changes in DNA methylation. Kobe J Med Sci 54:E241-E249

Oliver P, Reynes B, Caimari A, Palou A (2013) Peripheral blood mononuclear cells: a potential source of homeostatic imbalance markers associated with obesity development. Pflug Arch 465:459-468

Petrov PD, Ribot J, Palou A, Bonet ML (2015) Improved metabolic regulation is associated with retinoblastoma protein gene haploinsufficiency in mice. Am J Physiol Endocrinol Metab 308:E172-E183

Reynes B, Diaz-Rua R, Cifre M, Oliver P, Palou A (2015) Peripheral blood mononuclear cells as a potential source of biomarkers to test the efficacy of weight-loss strategies. Obesity (Silver Spring) 23:28-31

Scully T (2014) Obesity. Nature 508:S49

Takahashi M, Kamei Y, Ezaki O (2005) Mest/Peg1 imprinted gene enlarges adipocytes and is a marker of adipocyte size. Am J Physiol Endocrinol Metab 288:E117-E124

Voigt A, Agnew K, van Schothorst EM, Keijer J, Klaus S (2013) Short-term, high fat feeding-induced changes in white adipose tissue gene expression are highly predictive for long-term changes. Mol Nutr Food Res 57:1423-1434

Zhang Y, Proenca R, Maffei M, Barone M, Leopold L, Friedman JM (1994) Positional cloning of the mouse obese gene and its human homologue. Nature 372:425-432 